25 (least squares [LS] mean [95\% CI]: Positive, $-7.0[-8.1,-6.0]$; Negative, $-3.7[-4.7,-2.8]$; General, $-11.1[-12.7,-9.5])$, as did CGI-S scores (LS mean [95\% CI] change at week 25: - 1.2 [-1.4-1.0]). Caregiver burden decreased over the treatment period, with the largest decline noted at week 9 for AL patients' caregivers (mean change from baseline at week 9: -8.4 ; week $25:-8.9$ ). Over weeks 5,9 , and $17,70.8 \%-74.7 \%$ of AL-treated patients were somewhat or very satisfied with treatment. Mean Q-LES-Q-SF total scores were stable. With PP, PANSS subscale and CGI-S scores improved from baseline to study end (LS mean [95\% CI] changes at week 25: Positive, $-7.1[-8.2,-5.9]$; Negative, $-3.5[-4.6,-2.5]$; General, -10.4 $[-12.1,-8.6]$; CGI-S, $-1.2[-1.5,-1.0])$. Mean caregiver burden decreased (week 9: -8.8 ; week 25: -9.2). Most PP patients were satisfied or very satisfied with treatment $(64.7 \%-69.3 \%$ at weeks 5,9 , and 17), and mean Q-LES-Q-SF total scores were stable.

Conclusion. In ALPINE, patients who initiated AL or PP in the hospital and continued treatment during outpatient care experienced improvement in schizophrenia symptoms and reported satisfaction with medication, decreased caregiver burden, and stable quality of life.

Funding. Alkermes, Inc.

\section{Treating Comorbid Childhood Bipolar Disorder and ADHD}

\author{
Darlyne G. Nemeth, PhD, MP, MPAP, \\ Kayla M. Chustz, BS and Cody M. Capps, BS
}

Neuropsychology Center of Louisiana, Baton Rouge, LA, USA

Presenting Author: Cody Capps

\begin{abstract}
Objectives. Pediatric mania is difficult to distinguish from childhood hyperactivity. Both share 3 common symptoms: distractibility, motoric hyperactivity, and talkativeness. Oftentimes, children are referred from their pediatrician due to a lack of appropriate response to stimulant medication. Pediatricians have learned that merely raising the dose or changing the stimulant does not work. A thorough neuropsychological evaluation often reveals bipolar mania. They may have comorbid bipolar disorder and ADHD. This poster paper will examine measures that can assist in this important differential diagnosis as well as offer treatment options, including medication management.

Methods. This case study includes three pediatric patients diagnosed with childhood bipolar disorder and ADHD. A comprehensive psychoeducational assessment was conducted for each of the patients, which resulted in this comorbid diagnosis.

Results. One of the most helpful measures was the TOVA (i.e., Test of Variables of Attention). When a child's attention and impulsivity scores are normal, and response time and variability scores are abnormal, both on and off medication, that is an indication of a mood disorder, These children also performed poorly on measures of processing speed, and verbal learning and interference tasks. Measures of affect and personality were important diagnostically. A combination of amantadine and either clonidine HCL ER or propranolol, as prescribed by a medical
\end{abstract}

psychologist, were found to be effective in controlling the symptoms of this comorbid diagnosis.

Conclusions. An evaluation of children's intellectual, attentional, behavioral, mood, and personality functioning is crucial for a differential diagnosis. In cases of comorbidity, ADHD and childhood bipolar disorder, the sooner the child is on appropriate medications, the better. When just the surface diagnosis of ADHD is medicated, the outcome is often problematic. There may be a poor response to treatment and a higher rate of suicide.

\section{Hospitalization Risk Among Adults with Bipolar I Disorder Treated with Oral Atypical Antipsychotics: A Long-Term Data Analysis of Medicaid Claims Data}

\author{
Xiaoli Niu, $\mathrm{PhD}^{1}$, Phani Veeranki, MD, $\mathrm{DrPH}^{2}$, \\ Syvart Dennen, $\mathrm{MS}^{2}$, Yanmei Liu, MS ${ }^{2}$, \\ Jason Shafrin, $\mathrm{PhD}^{2}$, Carole Dembek, $\mathrm{MS}^{1}$, \\ Kimberly Laubmeier, $\mathrm{PhD}^{1}$ and G. Rhys Williams, $\mathrm{DSc}^{1}$
}

${ }^{1}$ Sunovion Pharmaceuticals Inc., Marlborough, MA, USA, and ${ }^{2}$ PRECISIONheor, Los Angeles, CA, USA

Presenting Author: Kimberly Laubmeier

\begin{abstract}
Objective. To compare the risk of hospitalization for adult Medicaid beneficiaries with bipolar I disorder (BPD-I) when treated with lurasidone compared to other atypical antipsychotics (AAPs) as monotherapy.

Methods. Using IBM MarketScan Multi-State Medicaid Claims database, a retrospective cohort study was conducted on adult BPD-I patients who initiated an AAP (index date) between January 1, 2014 and June 30, 2019. Patients were required to be continuously enrolled during the 12 -month pre- and 24-month post-index date. Marginal structural models were performed to estimate the risk of hospitalization (all-cause, BPD-I-related, and psychiatricrelated) associated with each AAP and the average length of stay. Results. The analysis included 8262 adult BPD-I patients, of whom AAP use was divided between lurasidone (14\%), aripiprazole (17\%), olanzapine ( $8 \%)$, quetiapine (29\%), risperidone (10\%), no/minimal (1\%) or other $(21 \%)$ during each month of post-index period. The adjusted odds ratios (aORs) for all-cause hospitalization were significantly higher for olanzapine $(\mathrm{aOR}=1.60,95 \% \mathrm{CI}=1.09-2.10)$ and quetiapine $(\mathrm{aOR}=1.54,95 \% \mathrm{CI}=1.18-1.89)$, compared to lurasidone. The aORs for BPD-I-related hospitalization were significantly higher for quetiapine $(\mathrm{aOR}=1.57,95 \% \mathrm{CI}=1.10-2.04)$ and risperidone ( $\mathrm{aOR}=1.80,95 \% \mathrm{CI}=1.04-2.56)$ compared to lurasidone. The average length of hospital stay was more than twice as high for quetiapine compared to lurasidone $(\mathrm{aRR}=2.12,95 \% \mathrm{CI}=1.32-2.92)$. The risk of psychiatric-related hospitalization was numerically lower for lurasidone compared to all other AAPs.
\end{abstract}

Conclusion. Over a 24-month follow-up period, lurasidonetreated adult BPD-1 patients had significantly lower risk of all- 
cause hospitalization than those treated with olanzapine and quetiapine, lower risk of BPD-I-related hospitalization than quetiapine and risperidone, and fewer hospital days than quetiapine in a Medicaid population.

Funding. Sunovion Pharmaceuticals Inc.

\section{Discovering Non-Invasive Biomarkers Predictive of Opioid Use Disorder Treatment Response}

Amir Levine, $\mathrm{MD}^{1}$, Kelly Clemenza, $\mathrm{MS}^{1}$,
Shira Weiss, $\mathrm{HBSc}^{1}, \mathrm{Adam}^{1}$ Bisaga, $\mathrm{MD}^{1}$, Erez Eitan, $\mathrm{PhD}^{2}$,
Maria Carla Gerra, $\mathrm{PhD}^{3}$, Claudia Donnini, $\mathrm{PhD}^{3}$,
Gilberto Gerra, $\mathrm{MD}^{4}$ and Benjamin Garcia, $\mathrm{PhD}^{5}$

${ }^{1}$ Columbia University, New York, NY, USA, ${ }^{2}$ NeuroDex Incorporated, Natick, MA, USA, ${ }^{3}$ University of Parma, Parma, Emilia-Romagna, Italy, ${ }^{4}$ United Nations Office on Drugs and Crime, Vienna, Austria, and ${ }^{5}$ University of Pennsylvania, Philadelphia, PA, USA

\section{Presenting Author: Kelly Clemenza}

liquid chromatography-mass spectrometry. EV miRNA cargo was determined by RNA sequencing.

Results. We found one lipid class and six miRNAs that differed significantly between the naltrexone group and the methadone and control groups. We also found that histone H3acK9acK14 was increasingly acetylated in PMBCs from both the methadone and naltrexone groups compared to controls.

Discussion. Naltrexone, which is used in treatment of OUD and other substance use disorders as well as disorders of impulse control, was found to have multiple potential corresponding molecular signatures that can be identified after long-term treatment. It remains to be seen if these markers can also be a good predictor for treatment response. In addition, significant gender differences in EV content are found between men and women with OUD, which supports the importance of examining changes in response to treatment in a gender informed way.

\section{Could Improved Microcirculation Reverse the Effects of Fetal Alcohol Syndrome? A Review}

\author{
Arman Phillipe Yavari, BA
}

University of Colorado at Boulder, Boulder, CO, USA

Presenting Author: Arman Phillipe Yavari

\begin{abstract}
Fetal alcohol spectrum disorders, (FASDs) are a spectrum of neurodevelopmental and cognitive conditions, caused by the consumption of alcohol in varying amounts in utero that affect an estimated $1-5 \%$ of the general population in the United States. The effects of the spectrum of disorders are lifelong, resulting in often significant learning difficulties, cognitive deficits, and behavioral issues. It is known that blood flow and microcirculation to the brain are affected by the consumption of alcohol in utero, and that microcirculation and neurology are intimately linked, with changes in the former having drastic effects on the latter. The purpose of the current review is to analyze whether improvement in microcirculation in the infant or child with FASDs could affect quality of clinical outcomes in the spectrum of disorders, or lead to the improvement of disorderrelated symptoms. Scientific evidence from the literature suggests that, in theory, improving cerebral microcirculation in the infant or young child with FASDs could lead to a corresponding improvement in neurological health, and potentially an improvement in cerebral development, which may in theory lead to a lessening of symptoms. Further review into the connection between microcirculation and neurological health, and correspondingly, clinical outcomes in patients with neurological deficits, is warranted.
\end{abstract}

